\title{
Adapter les zones côtières aux changements climatiques : de la consultation à l'action
}

\author{
Jean-Pierre SAVARD et Alain Bourque
}

Ouranos. 550 Sherbrooke ouest, $19^{\text {ième }}$ étage, tour ouest, Montréal, QC. H3A

1B9 Tél: 11 (514) 282-6464 Fax: 11 (514) 282-7131

e-mail: savard.jean-pierre@ouranos.ca

\begin{abstract}
Résumé :
La population riveraine de la région du Golfe du Saint-Laurent (GSL) du Québec est vulnérable à l'impact des changements climatiques (CC) sur l'érosion côtière. La hausse du niveau de la mer et les modifications de la fréquence des tempêtes, du régime des glaces, des cycles de gel et d'autres extrêmes climatiques affectent l'érosion des berges. Un projet d'étude de la zone littorale a été amorcé par Ouranos en 2005 afin de faciliter l'adaptation des communautés côtières de la région $d u$ GSL aux CC. L'équipe de recherche a utilisé une approche multidisciplinaire fortement intégrée permettant d'étudier les liens entre le climat régional, les conditions océanographiques et l'évolution de la zone littorale. De plus, l'équipe de projet a testé une méthode participative innovatrice permettant d'acheminer vers les décideurs clés de la zone côtière les points de vue d'une vaste coalition de groupes et d'organisations ayant des intérêts ou des activités dans cette zone, ainsi que les informations scientifiques pertinentes à la prise de décision. Les avantages de cette approche sont : la prise en compte de la science du climat dans la gestion de la zone côtière, une amélioration des communications entre les chercheurs et les acteurs et décideurs de la zone côtière, le développement d'une capacité d'intégrer les connaissances scientifiques à l'échelle régionale et un contexte favorisant l'exécution plus rapide des solutions d'adaptation.
\end{abstract}

\begin{abstract}
:
The population of the maritime region of Quebec is vulnerable to coastal erosion that will be affected by climate change (CC). Sea level rise, extreme events such as storms and intense rainfall, ice climate, thaw and frost cycles are all climate variables that can increase erosion and affect coastal infrastructures. In 2005, a research project was initiated to assess impacts of CC on the coastal zone and to facilitate the adaptation process of coastal communities. The project team addressed the issue though a multidisciplinary and integrated approach allowing a complete analysis of various interactions between changes in regional climate, oceanic conditions and recent evolution of the coastal zone. In addition to studying the physical aspect of this issue, a participative method of adaptation process selection was tested. This method was applied at three test sites selected on the basis of their specific coastal typology and human activities. The participative process was designed to facilitate the transfer of multi-dimension information, socio economic, environmental, technical and scientific, to the policy makers and deciders. Project benefits include the following: inserting science of climate and coastal dynamics in the decision process, improving communications between stake holders, researchers and decision makers, developing a core of local and regional expertise on the issue and accelerating the implementation of adaptation solutions.
\end{abstract}

Mots-clés : Environnement littoral -- Adaptation changements climatiques -- Génie côtier-Méthodes participatives 


\section{$1 \quad$ Introduction}

Cette étude, amorcée en septembre 2005, avait pour objectif principal d'évaluer les principaux impacts appréhendés des changements climatiques (CC) sur la dynamique côtière dans le golfe du Saint-Laurent (GSL) et de proposer aux pouvoirs publics des solutions d'adaptation visant à réduire la vulnérabilité des populations côtières. Bien que l'étude porte sur l'ensemble du GSL, une partie importante de l'effort de recherche a été ciblée sur trois sites témoins, soit Sept-Îles, Percé et les Îles-de-la-Madeleine (figure 1).

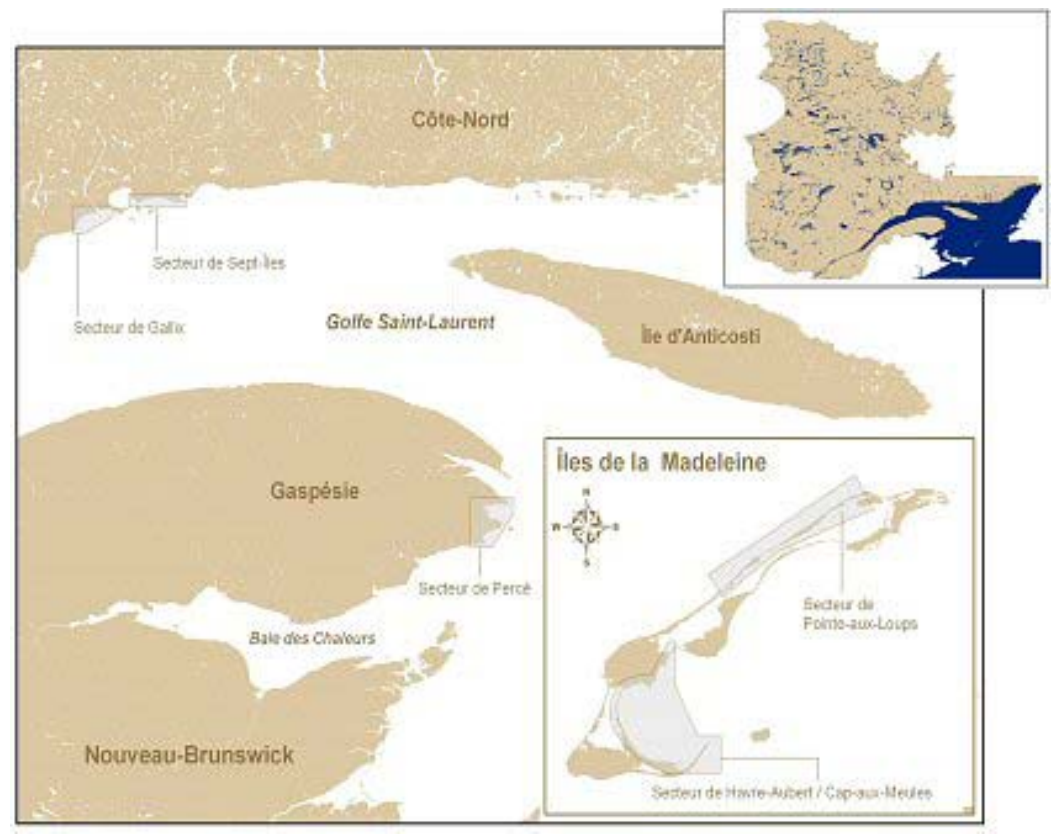

FIGURE 1 Le golfe du Saint-Laurent et les trois sites témoins

La région maritime du Québec occupe une superficie de $450000 \mathrm{~km}^{2}$. La population essentiellement côtière est dispersée sur une bande riveraine de $4000 \mathrm{~km}$ de longueur. Les trois sites témoins ont été choisis parce qu'ils sont une bonne représentation des diverses conditions socio-économiques et environnementales de cette région. La structure organisationnelle de l'étude est présentée à la figure 2. L'équipe de travail a été divisée en trois groupes. Le premier a mené les recherches sur le climat, l'océanographie et les conditions de glace; le second s'est penché sur la dynamique côtière, les vulnérabilités socio-économiques et les risques; le troisième est constitué d'intervenants locaux et régionaux de la zone côtière regroupés en trois comités (un par zone témoin) qui ont collaboré à l'étude en évaluant divers scénarios illustrant l'influence appréhendée des changements climatiques. Ces comités de concertation ont été constitués lors d'ateliers 
tenus au début de l'étude. Ces ateliers regroupaient des représentants d'une vaste gamme d'organisations locales et régionales de la société civile et des diverses instances gouvernementales pouvant apporter des points de vue sur la gestion de la zone côtière.

L'originalité de la présente étude tient à la méthodologie utilisée pour évaluer l'impact des changements climatiques sur la zone côtière et à la méthode participative dynamique utilisée pour former ces comités d'usagers intégrés au projet de recherche, signataires du rapport de recherche et jouant un rôle décisif dans la recherche des solutions d'adaptation.

\section{$2 \quad$ Méthode participative dynamique}

L'adaptation des communautés côtières aux changements climatiques (CC) se heurte généralement à deux obstacles majeurs, soit : 1) l'élaboration de scénarios quantitatifs de l'impact des CC sur la zone côtière et 2) le transfert vers les décideurs et les utilisateurs des connaissances nécessaires à une action concertée. La seconde difficulté est particulièrement importante lorsque les problématiques liées au CC sont complexes comme c'est le cas en zones côtières.

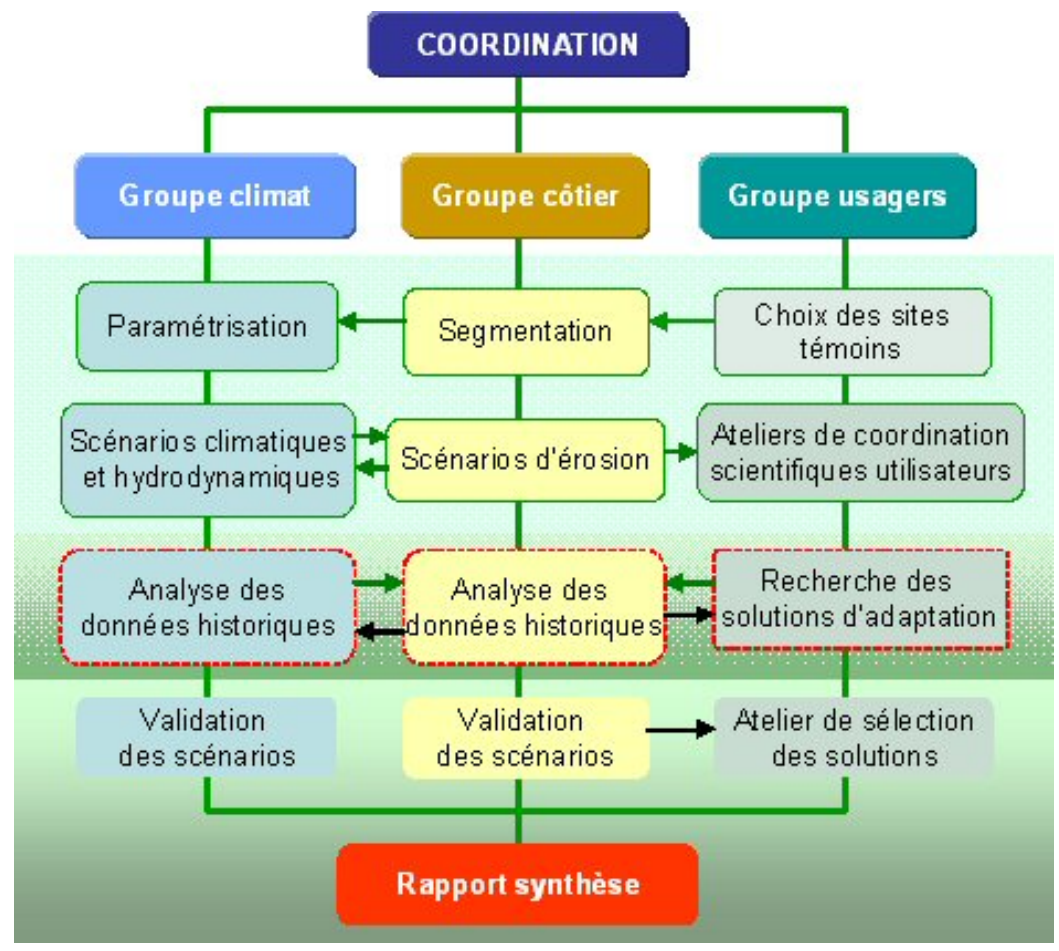




\subsection{Le chemin vers l'adaptation}

L'arrimage de la connaissance scientifique, en constante mutation, et du processus de concertation indispensable à une prise de décision à la fois équitable, durable et efficace est un problème qui a fait l'objet de nombreuses études (Füssel 2007, Vogel et al. 2007). Dans la zone côtière, plusieurs études théoriques ont été réalisées (van Aalst et al. 2007) et diverses expériences de participation sont menées. Cependant, les tentatives d'intégration verticale complète sont rares. Le plus souvent, la recherche sur le milieu physique, le climat et le régime côtier sont effectuées d'un côté par des chercheurs spécialisés et les expériences de participation sont réalisées séparément par des chercheurs en géographie humaine ou en sociologie ou lors de forums citoyens et d'assemblées publiques ou semi publiques tenues par des organisations impliquées dans la gestion de la zone côtière.

L'adaptation des communautés côtières est généralement le résultat d'une concertation plus ou moins poussée entre les décideurs locaux ou régionaux et ceux des instances gouvernementales plus centrales (provinciale et fédérale). Ces décideurs doivent composer avec un grand nombre d'enjeux croisés qui ne relèvent pas nécessairement de la science du milieu physique (climat et dynamique côtière). Très souvent, la science est loin d'être le principal facteur influençant une décision.

La figure 3 illustre schématiquement l'ensemble des éléments à prendre en compte pour que l'adaptation aux changements climatiques soit fondée une concertation des acteurs clefs du milieu. Celle-ci illustre schématiquement les trois axes de concertation nécessaires à la prise de décision, soit l'axe scientifique, l'axe de gouvernance et l'axe sociétal. Dans l'axe scientifique, on retrouve les principales disciplines scientifiques impliquées, c'est à dire les sciences du climat, l'océanographie, la géographie et la géomorphologie côtières jusqu'à l'ingénierie civil côtière. Dans l'axe sociétal, on retrouve les grandes tendances typiquement représentées par la dichotomie gauche droite, avec d'un côté les points de vue environnementaux et communautaires (sauvegarde de l'environnement et des écosystèmes côtiers, préservation de paysages naturels, équité et justice sociale) et de l'autre, les points de vue dits «de droite» dont le développement économique, le commerce, l'industrie, la propriété privée, etc. Enfin, l'axe de gouvernance ou politique comporte tous les paliers hiérarchiques des fonctions publiques et les divers niveaux de gouvernement (central, provincial, régional et municipal). 


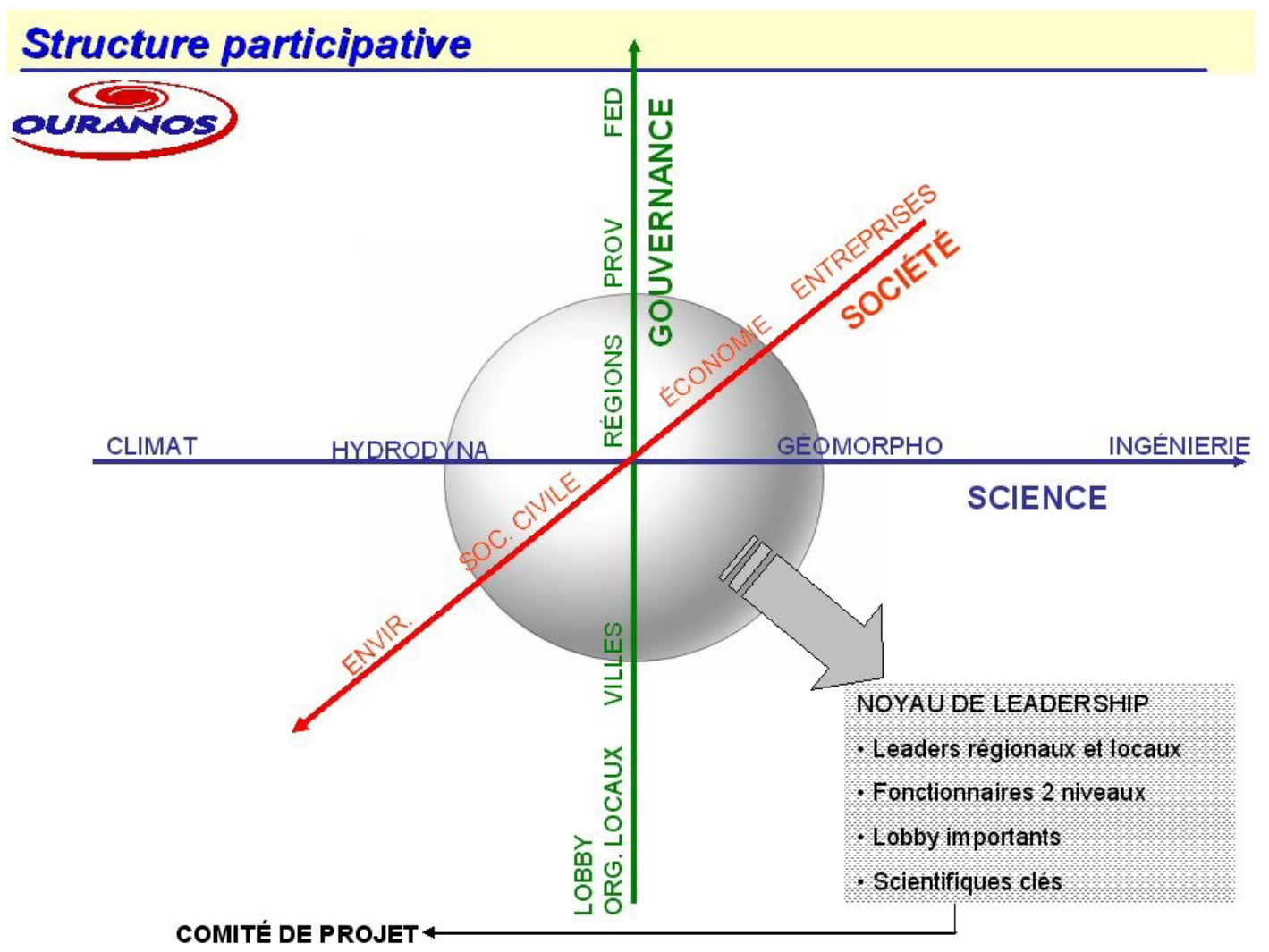

Figure 3 Représentation des axes dominants d'intervention dans le processus décisionnel en zone côtière et de la formation d'un noyau de leadership.

Les décisions d'adaptation aux changements climatiques sont prises au niveau de l'axe vertical (axe de gouvernance) par des décideurs élus ou nommés. La partie supérieure de cet axe est occupée par les décideurs des instances nationales (au Canada, ce sont les gouvernements fédéraux et provinciaux) alors que la partie inférieure regroupe les décideurs des instances locales (municipalités, administrations régionales et divers lobbies, incluant des individus).

Les décideurs oeuvrant au niveau national ont une vision globale des problématiques côtières (portrait d'ensemble) et peuvent prioriser les besoins de cette zone par rapport à l'ensemble des priorités socio-économiques ou autres (santé, éducation, transport, etc.). Ils ont aussi accès à l'expertise et à la 
connaissance scientifique disponible parce qu'ils peuvent en payer le coût, ce qui n'est pas toujours le cas des décideurs locaux. Par contre, il est difficile, pour un décideurs d'une instance nationale de comprendre en détail tous les enjeux locaux et régionaux liés à la gestion des zones côtières. En général, les décideurs nationaux ont tendance à adopter des politiques, des programmes, des législations et des règlements qui s'appliquent de manière assez uniforme. Cette approche topdown permet une certaine harmonisation des actions mais elle manque de souplesse et les décisions top-down sont souvent très mal comprises et mal acceptées par les communautés et leurs représentants locaux.

Il existe une grande variété de formes de concertation publique du «milieu» qu'on peut regrouper sous l'étiquette «bottom-up ». En général, elles visent à présenter les problématiques de gestion côtière de manière à ce que l'opinion des principaux intéressés soit exprimée, débattue, consignée et transmise aux gestionnaires des instances nationales. Parfois, ce genre de concertation aboutit à des actions directes à l'échelle locale qui se réalisent sans que les instances nationales soient consultées.

En théorie, les approches «bottom-up», qui s'appuient sur une concertation intensive du «milieu», ont de meilleures chances d'engendrer des décisions acceptées par les communautés concernées. Cependant, les instances locales sont souvent très mal équipées pour insérer la science côtière dans le processus de décision et les modes de concertation sont parfois déficients. Les décideurs locaux risquent d'être sensibles aux lobbies les plus puissants ou les plus articulés et de négliger complètement une bonne partie des éléments illustrés à la figure 3 . Enfin, la zone côtière est tellement propice aux conflits d'usage que les visions contradictoires sont très nombreuses et la concertation, lorsqu'elle est mal organisée, risque de favoriser la confrontation plutôt que l'émergence de consensus.

\subsection{La méthode participative : le processus}

Dans le cadre de l'étude, nous avons expérimenté un processus de concertation ayant pour but de rapprocher les instances décisionnelles locales et nationales en favorisant une mise à niveau des deux groupes au plan scientifique. Dans un premier temps, on a demandé aux chercheurs des groupes climat et côtier (voir figure 2) d'accepter une méthodologie de travail très intégrée permettant de couvrir l'ensemble des éléments clés reliant le climat et l'érosion côtière. Cette 
façon de faire n'est pas très courante, les chercheurs ayant plutôt tendance à isoler certains paramètres et à les explorer en profondeur.

Dans un deuxième temps, nous avons constitué des comités de concertation formés de personnes représentant une large gamme de points de vue sur la zone côtière. Ces personnes ont été intégrées à l'équipe scientifique en ayant pour mission d'évaluer les options d'adaptation et de proposer les recommandations qui apparaissent dans le rapport. L'équipe scientifique a donc accepté de jouer un rôle de soutient et de laisser à des non scientifiques la responsabilité de déterminer les meilleures options d'adaptation proposées dans ce rapport.

Pour éviter que le comité de concertation passe la majeure partie du projet à attendre les résultats de l'équipe de recherche, ce comité a commencé son travail en se basant sur des scénarios simplifiés de type "what if » qui permettent d'évaluer la vulnérabilité à des hypothèses plausibles sur les taux d'érosion qui prévaudront dans un climat futur. Cependant, à mesure que le projet avance, les scénarios d'érosion sont de plus en plus rattachés aux processus climatiques et finalement, l'équipe de recherche vise à identifier les scénarios considérés comme les plus probables et à quantifier le risque. L'élément primordial pour que cette façon de procéder converge vers une véritable étude d'impact et d'adaptation des communautés côtières au changements climatiques est que les scénarios d'érosion utilisés en parallèle par l'équipe scientifique et le comité de concertation soient les mêmes.

Les comités d'usagers ont été constitués lors d'ateliers organisés en début de projet dans chacune des trois zones témoins de l'étude. L'organisation de ces ateliers devait se plier à quelques règles simples mais importantes pour assurer bon déroulement du projet :

1) l'atelier était constitué de représentants d'organisations identifiées à la figure 3 . Aucune organisation impliquée dans la zone côtière n'est exclue à priori, mais les individus ne représentant qu'eux-mêmes ne sont pas admis;

2) l'organisme qui invite les participants à l'atelier doit avoir une réputation de neutralité bienveillante, d'objectivité et de crédibilité dans le dossier ; dans ce cas, Ouranos a joué ce rôle parce que cette organisation est un organisme indépendant sans but lucratif, voué à la recherche sur l'impact et l'adaptation aux changements climatiques. Ouranos n'est pas directement impliqué dans les contentieux pouvant 
diviser certains ministères du gouvernement et des municipalités ou des organismes du milieu.

3) les participants locaux et régionaux invités à l'atelier occupaient généralement une fonction de direction pour l'organisation qu'ils représentent alors que les participants provenant des gouvernements centraux occupaient un niveau intermédiaire comprenant généralement deux types de fonctions, soit du service direct à l'échelle locale et des fonctions administratives ou de gestion à l'échelle de leur organisation.

4) à chaque atelier, d'une durée d'une journée, l'ordre du jour comportait trois items, soit une courte présentation du projet d'étude par les scientifiques du projet (environ 1 h30 heures au total), une revue des enjeux liés à la zone littorale et aux changements climatiques; les participants étaient invités à identifier les enjeux prioritaires de l'étude. Enfin, le troisième point à l'ordre du jour était la formation du comité de concertation et le choix des présidents de ce comité. On expliquait aux participants le rôle du comité, soit un petit groupe de 8 à 10 personnes représentant les organisations clés de la zone côtière et une variété de point de vue sur la gestion de ce milieu qui seraient responsables du contenu du rapport final portant sur les solutions d'adaptation.

La réponse à l'invitation aux ateliers a été supérieure à $80 \%$ à chacun des trois sites. Dans tous les cas, la plupart des organisations clés, comme la municipalité, les municipalités régionales de conté, les comités ZIP (organismes d'action communautaire régionale en environnement) et des associations de riverains étaient présents. Les gouvernements centraux fédéraux et provinciaux ont délégués des représentants en environnement, en sécurité publique, en transport, en pêche de même que pour les parcs, le tourisme et les affaires municipales. Le secteur privé a moins bien répondu à l'appel, mais certaines associations de pêcheur et associations touristiques ont délégué des représentants.

Les ateliers se sont tous déroulés tel que prévu. Les priorités assignées par les participants ont aidé l'équipe de recherche à cibler les enjeux les plus importants. Les participants ont généralement beaucoup apprécié le fait qu'une équipe de recherche les intègre dans le projet de recherche. Les comités ont été constitués par les assemblées et les présidents de comités ont aussi été nommés par les participants.

Les membres des trois comités se sont réunis à deux ou trois reprises dans chaque 
zone témoin. Chaque rencontre des membres des comités se déroulait de la manière suivante : d'abord, les représentants de l'équipe scientifique fournissaient un bref compte-rendu de l'état d'avancement des recherches liées au projet. On passait ensuite le reste de la journée à examiner les scénarios préparés par l'équipe de dynamique côtière afin d'évaluer les solutions d'adaptation possible. Ces scénarios sont décrits ci-dessous.

Tableau 1. Scénarios d'érosion utilisés pour cartographier l'évolution du trait de côte jusqu'en 2050

\begin{tabular}{|c|c|}
\hline Scénarios pour 2050 & Description \\
\hline $\begin{array}{l}\text { s1 : taux de déplacement moyen } \\
\text { de la ligne de rivage entre } \\
1931 \text { et } 2006\end{array}$ & $\begin{array}{l}\text { Ce scénario suppose que l'effet } \\
\text { des changements climatiques ne } \\
\text { modifiera pas les taux moyens de } \\
\text { recul des talus côtiers d'ici } \\
2050 \text {. }\end{array}$ \\
\hline $\begin{array}{l}\text { S2 : taux d'érosion moyen } \\
\text { mesuré pour un intervalle de } 10 \\
\text { à } 15 \text { ans où l'érosion a été la } \\
\text { plus intense lors de la période } \\
\text { 1931-2006 }\end{array}$ & $\begin{array}{l}\text { Ce scénario considère comme } \\
\text { probable une accélération de } \\
\text { l'érosion côtière en raison des } \\
\text { changements climatiques. }\end{array}$ \\
\hline $\begin{array}{l}\text { s3 : Taux moyen des valeurs } \\
\text { supérieures à la moyenne des } \\
\text { taux de recul pour un } \\
\text { intervalle de } 10 \text { à } 15 \text { ans où } \\
\text { l'érosion a été la plus intense } \\
\text { lors de la période } 1931-2006\end{array}$ & $\begin{array}{l}\text { Ce scénario considère comme } \\
\text { probable une accélération très } \\
\text { élevée de l'érosion côtière en } \\
\text { raison des changements } \\
\text { climatiques et de facteurs } \\
\text { anthropiques aggravants. }\end{array}$ \\
\hline
\end{tabular}

L'équipe de dynamique côtière a préparé des cartes sur photos aériennes orthorectifiées et présenté les scénarios en illustrant la position probable de la ligne de rivage selon chacun des trois scénarios d'érosion ou d'évolution de la côte (figure 4). Cette présentation simple permettait de visualiser la position du talus côtier en 2050 pour chacun des trois scénarios pour chaque segment de berge cartographié (la cartographie portait sur les zones identifiées comme prioritaires lors de l'atelier du début de projet). Les scénarios S1, S2 et S3, tous tirés des taux historiques d'érosion, sont les mêmes pour l'équipe de recherche (groupes 1 et 2) que pour les comités de concertation (groupe 3). Le travail de l'équipe de recherche est de déterminer lequel des trois scénarios est le plus plausible compte tenu des CC alors que celui des comité est d'évaluer les solutions d'adaptation les plus appropriées pour tous les scénarios. 


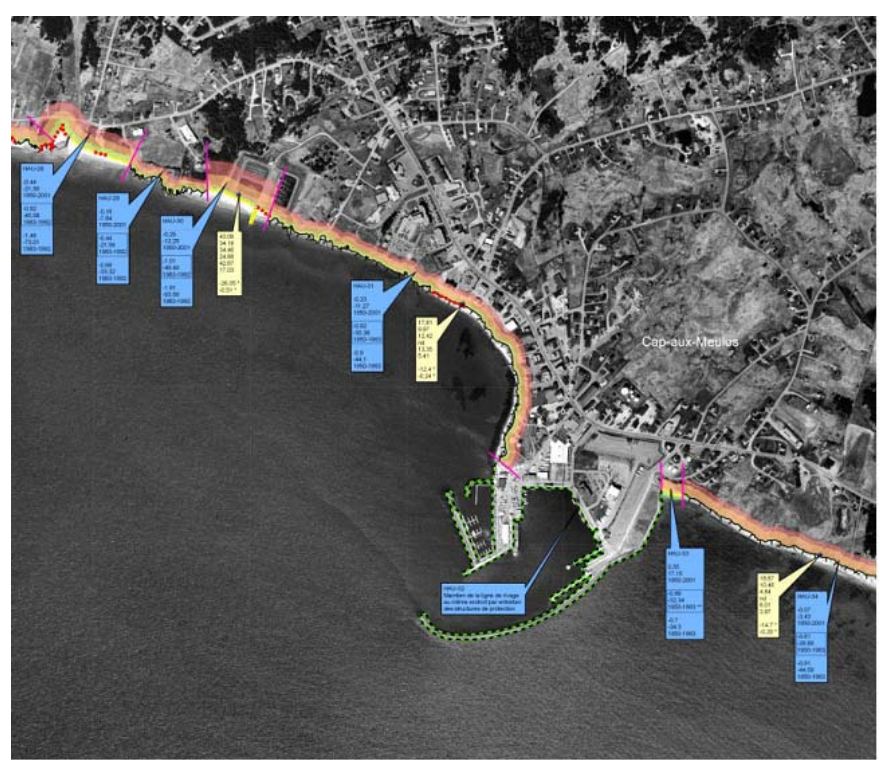

FIGURE 3 Exemple de cartographie numérisée des scénarios S1, S2

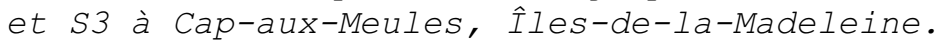

La cartographie des scénarios d'érosion a permis aux membres des comités de visualiser sur les photographies les infrastructures (routes, maisons, bâtiments, parcs, plages etc.) menacés par l'érosion d'ici une quarantaine d'année (période de projection considérée comme raisonnable). On leur demandait alors de suggérer les solutions et les scientifiques du projet leur fournissaient tout l'appui technique possible se rattachant aux diverses solutions possibles. Si les scientifiques n'avaient pas la réponse à une question des participants, cette question était prise en délibéré et l'on essayait d'apporter une réponse à la prochaine rencontre. Dans certains cas, à la demande des participants, les municipalités et certains ministères représentés au sein des comités ont lancé des études complémentaires en cours de projet afin de répondre à certaines questions.

Deux points importants méritent d'être soulignés. L'analyse des solutions par les comités est basée sur la recherche de consensus. Les participants examinent les segments de berge homogènes de leur zone témoins sur les cartes pour chacun des trois scénarios. S'ils s'entendent rapidement entre eux sur la meilleure solution pour cette berge et ce scénario, ce choix est noté et consigné dans le rapport du comité. S'ils ne s'entendent pas sur la solution d'adaptation à recommander, on essaie alors d'établir les points de divergences ou d'incertitude et de formuler des questions permettant de résoudre ces problèmes et on passe à un autre segment de berges ou un autre scénario. Autrement dit, on règle d'abord les questions qui font rapidement consensus (il y en a toujours un certain nombre) et on reprend à la rencontre suivante les questions en litige en ajoutant plus d'information technique. 
Cette progression par consensus est un élément important de la réussite des rencontres parce qu'elle permet aux participants de développer un esprit d'équipe, de se faire confiance les uns aux autres et d'obtenir des accords ou des succès en commun. Il ne faut pas oublier que l'équipe de projet ne choisit pas les membres des comités et certains d'entre eux peuvent avoir des positions très antagonistes ou même hostiles après des années de litiges et de conflits d'usages. Cette manière d'aborder les questions a eu pour résultats que les groupes formés se sont maintenus (pas d'abandon) pendant tout le projet et que des consensus assez nets ont été obtenu sur la très grande majorité des points abordés malgré la complexité de certains problèmes et souvent, en dépit d'importantes divergences de point de vue au départ.

Le second point important à souligner est la différence entre cette méthode de concertation et des processus de consultation ou de négociation bipartites ou multipartites souvent confondus avec le noyau de leadership par les organismes centralisés. Une rencontre entre des scientifiques ou experts, entre des fonctionnaires d'un gouvernement central et les dirigeants d'une municipalité peut ressembler à un mécanisme participatif dynamique, mais ce n'est pas le cas. Dans ce type de concertation, seuls des décideurs et leurs représentants sont invités, souvent par une approche top down. De nombreux points de vue sont exclus d'emblée parce que les personnes ou les organisations pouvant les exprimer ne sont pas invitées à les présenter ou à les débattre. Les intérêts d'une municipalité ne sont pas automatiquement les mêmes que ceux des citoyens qu'elle est supposée représenter. Bref, il y a une différence fondamentale entre l'approche participative utilisée ici, où tous les participants sont choisis par le milieu sans exclusion préalable et une discussion multipartite. La recherche de consensus ne passe pas par l'élimination systématique des personnes qui font objection.

\section{$3 \quad$ Résultats}

Les résultats des recherches sur l'impact du climat sur les zones côtières des trois sites témoins et les solutions d'adaptation retenues par les comités de concertation du Groupe usagers du projet sont regroupés sous forme synthétique dans Savard et al. 2008 et disponibles en format .pdf sur le site suivant :

\section{http://www.ouranos.ca/doc/produit f.html}

Les comités ont proposé des solutions d'adaptation qui ont souvent surpris 
l'équipe de projet. A titre d'exemple de réussite, des propositions ont été soumises pour limiter les méthodes lourdes aux infrastructures critiques et impossibles à relocaliser telles que des hôpitaux, les routes essentielles non déplaçables ou autres. Le Ministère des Transport du Québec a accepté pour la première fois l'idée d'une recharge en sable assortie de méthodes légères (épis en pieux de bois ou équivalent) pour ralentir le transit sédimentaire dans certains secteurs pour protéger des routes. Cette décision a été suivie de négociations pour revaloriser le sable dragué par une industrie et par des essais pilotes. Les trois municipalités concernées par le projet ont révisé leurs schéma d'aménagement et repensé leur zonage afin d'éliminer la construction dans les secteurs à risques. A Sept-Îles, une coût avantage ont été menée pour évaluer les solutions proposées et finaliser les choix avant la mise en œuvres. Certaines discussions des comités ont donné lieu à des projets avant-gardistes de restauration de marais, de prise en charge de secteurs pour les transformer en zones protégées, de revalorisation de sable de dragage, etc. Les solutions d'adaptation recommandées par les membres des comités de concertation sont généralement des solutions durables au plan environnemental.

Tel que mentionné précédemment, l'un des points forts de la méthode a été de choisir les mêmes scénarios (S1, S2 et S3) d'érosion tant pour l'analyse des impacts des changements climatiques que pour celle de l'analyse des solutions. Le choix des scénarios présentés au tableau 1 est fondé sur l'hypothèse de travail selon laquelle les fluctuations décennales des taux d'érosion sont dues à des fluctuations de variables d'origine climatique qui contrôle la dynamique des berges. En comparant les taux d'érosion historiques à intervalle de l'ordre d'une décennie, on observe des fluctuations qui sont systématiquement comparées aux variables climatiques comme la fréquence des tempêtes causant de fortes vagues et des surcôtes sur la côte, la fréquence des cycles de gel et de dégel, la durée de la couverture de glace de mer, etc.

Ces variables climatiques ont fluctué dans le passé et les comparaison avec les taux d'érosion mesurés à partir de photographies aérienne ont permis d'identifier les mécanismes d'érosion prédominants. Les outils de la recherche sur le climat, principalement les modèles climatiques globaux et régionaux, ont alors été utilisés pour évaluer l'évolution future de ces variables clés jusqu'en 2050. Dans beaucoup de cas, on a constaté que l'ordre de grandeur des fluctuations climatiques observées à l'échelle d'une décennie depuis 1930 était similaire à celui des changements climatiques anticipés d'ici 2050. 
Cette méthode par analogie temporelle a permis à l'équipe scientifique de spécifier dans de nombreux cas le scénario le plus plausible parmi les trois scénarios d'érosion initiaux. Comme les membres des comités avaient examiné des solutions d'adaptation pour les trois scénarios, il leur a été possible de sélectionner le scénario probable et de recommander des actions spécifiques pour la plupart des segments de berge.

\section{$\underline{4 \quad \text { Conclusions }}$}

L'approche participative dynamique a permis d'atteindre le stade de la recommandation de solutions d'adaptation spécifiques au site dans les zones témoins du projet. Mais surtout, cette approche a permis d'amorcer la propagation des idées et des hypothèses de solution vers le sommet des organismes centralisé (gouvernements centraux) et vers la base des organismes du milieu (groupes de riverains, environnementaux, etc.) tout en développant un dialogue et une recherche de consensus au sein des principaux acteurs de l'adaptation, incluant les scientifiques.

L'approche participative a aussi permis aux recommandations formulées d'être mieux reçues par les décideurs des instances locales ou régionales, dont les trois municipalités visées par le projet pilote. Le dépôt du rapport synthèse le 4 juillet 2008 souligne ce résultat puisque ce rapport a été rendu public en présence et avec le support des trois maires des villes impliquées et de la Vice Première ministre du Québec. Le dépôt du rapport s'est réalisé conjointement avec l'annonce d'une chaire de recherche sur le littoral et d'un protocole d'entente entre le Ministère de la Sécurité publique du Québec et la municipalité des Îles-de-la-Madeleine sur la gestion du littoral.

Le fait d'intégrer des représentants locaux et régionaux dans un projet de recherche a été globalement bénéfique pour les chercheurs et les participants aux comités. La crédibilité des scientifiques du projet auprès des instances locales a augmenté, comme en témoigne les demandes formulées par les municipalités pour l'équipe de projet présente les résultats de l'étude aux conseils municipaux et aux citoyens des ces municipalités. Le travail de communication et d'explication aux communautés sera facilité par le fait que les participants locaux aux comités de concertation sont désormais mieux formés pour expliquer les choix et les décisions prises par ces comités aux autres organisations locales, aux citoyens et même aux médias. En fait, nous avons déjà pu constater que certains de ces participants attiraient l'attention des média parce qu'ils sont désormais en mesure 
d'expliquer de manière simple et nuancée les problématiques liées à l'érosion et aux changements climatiques.

L'approche a entraîné certaines retombées négatives inattendues. Par exemple, certaines autorités des gouvernements centraux ont perçu l'action des scientifiques du projet comme une menace parce qu'elle contribue à l'émergence d'un consensus régional quant aux solutions d'adaptation. Ce résultat est parfois perçu comme ayant contribué à former de puissants lobbies locaux qui sont maintenant mieux équipés pour forcer la main aux décideurs nationaux et les obliger à adopter les solutions proposées. Dans ce contexte, les scientifiques sont perçus comme ayant un parti pris envers les décideurs locaux. Ce résultat est assez surprenant parce que jusqu'à récemment, la méfiance envers les scientifiques était plutôt du côté des communautés côtières qui percevaient les chercheurs comme inféodés aux bailleurs de fonds gouvernementaux. Il est important que les scientifiques conservent une réputation de neutralité bienveillante et de recherche du bien commun, sans quoi le processus participatif, en dépit de ses avantages, ne sera par étendu à d'autres secteurs.

Le suivi du cheminement vers l'adaptation est un élément important du projet. Il est essentiel que les prochaines phases de recherche examinent les impacts du processus de concertation sur les processus décisionnel afin de s'assurer que la méthode produit des effets concrets et bénéfiques en terme d'adaptation.

\section{$5 \quad$ Références bibliographiques}

FUSSEL, H.-M (2007). Adaptation planning for climate change: concepts, assessment approaches, and key lessons. Sustainability science 2: 265-275.

GIEC (2007). Climate Change 2007: The Physical Science Basis. Contribution of Working Group I to the Fourth Assessment Report of the Intergovernmental Panel on Climate Change, SOLOMON, S., D. QIN, M. MANNING, Z. CHEN, M. MARQUIS, K.B. AVERYT, M. TIGNOR et H.L. MILLER (eds.), Cambridge (UK) et New York, NY, USA, Cambridge University Press.

SAVARD, J.-P., BERNATCHEZ, P., MORNEAU, F., SAUCIER, F., GACHON, P., SENVILLE, S., FRASER, C. ET JOLIVET, Y. (2008). Étude de la sensibilité des côtes et de la vulnérabilité des communautés du golfe du Saint-Laurent aux impacts des changements climatiques - Synthèse des résultats. Rapport d'Ouranos. 58 p. http://www.ouranos.caldoc/produit f.html 
Xèmes Journées Nationales Génie Côtier - Génie Civil, 14-16 octobre 2008, Sophia Antipolis

VAN AALST, M. K., et T. CANNON et al. (2008). Community level adaptation to climate change: The potential role of participatory community risk assessment. Global Environmental Change 18(1): 165-179.

VOGEL, C., S. C. et MOSER et al. (2007). Linking vulnerability, adaptation, and resilience science to practice: Pathways, players, and partnerships. Global Environmental Change 17(3-4): 349-364. 
Changement climatique

502 